\title{
A note on global regularity in optimal transportion
}

\author{
Neil S. Trudinger
}

Received: 5 August 2013 / Revised: 10 October 2013 / Accepted: 11 October 2013 /

Published online: 23 October 2013

(C) The Author(s) 2013. This article is published with open access at SpringerLink.com

\begin{abstract}
We indicate how recent work of Figalli-Kim-McCann and Vetois can be used to improve previous results of Trudinger and Wang on classical solvability of the second boundary value problem for Monge-Ampère type partial differential equations arising in optimal transportation together with the global regularity of the associated optimal mappings.
\end{abstract}

Keywords Optimal transportation · Monge-Ampère equations ·

Second boundary value problem

Mathematics Subject Classification (1991) Primary 35J66 · 35J96;

Secondary 49N60

\section{Introduction}

In this short note we use recent work of Figalli-Kim-McCann [4] and Vetois [24] on continuous differentiability and strict convexity of potentials in optimal transportation to improve previous results on global regularity of optimal mappings in [20]. The corresponding partial differential equations are Monge-Ampère type equations which have the general form:

Communicated by S.K. Jain.

Research supported by Australian Research Council Grant.

\footnotetext{
N. S. Trudinger $(\varangle)$

Centre for Mathematics and Its Applications, Australian National University,

Canberra, ACT 0200, Australia

e-mail: neil.trudinger@anu.edu.au
} 


$$
\operatorname{det}\left[D^{2} u-A(\cdot, u, D u)\right]=B(\cdot, u, D u),
$$

where $A$ and $B$ are given $n \times n$ matrix and scalar valued function defined on $\Omega \times \mathbb{R} \times \mathbb{R}^{n}$, where $\Omega$ is a domain in Euclidean $n$-space, $\mathbb{R}^{n}$. We use $(x, z, p)$ to denote points in $\Omega \times \mathbb{R} \times \mathbb{R}^{n}$ so that $A(x, z, p) \in \mathbb{R}^{n} \times \mathbb{R}^{n}, B(x, z, p) \in \mathbb{R}$. Equation (1.1) is elliptic with respect to a solution $u \in C^{2}(\Omega)$ whenever

$$
D^{2} u-A(\cdot, u, D u)>0
$$

whence also $B>0$.

We assume that $A$ is generated by a cost function $c \in C^{4}\left(\bar{\Omega} \times \bar{\Omega}^{*}\right)$ satisfying the following conditions, where $\Omega^{*} \subset \mathbb{R}^{n}$ is a target domain:

A1: The mappings $c_{x}(x, \cdot), c_{y}(\cdot, y)$ are one-to-one for each $x \in \Omega, y \in \Omega^{*}$;

A2: $\operatorname{det} c_{x, y} \neq 0$ in $\bar{\Omega} \times \bar{\Omega}^{*}$.

Conditions A1, A2 imply the existence of a $C^{3}$ mapping $Y$ given by $Y(x, p)=$ $c_{x}(x, \cdot)^{-1}(p)$ for $x \in \Omega$ and $p \in c_{x}(x, \cdot)\left(\Omega^{*}\right)$, with $Y_{p}=\left[c_{x, y}\right]^{-1}$. Moreover, for positive densities $f \in C^{0}(\Omega), g \in C^{0}\left(\Omega^{*}\right)$, the prescribed Jacobian equation,

$$
|\operatorname{det} D Y(\cdot, D u)|=f / g^{\circ} Y
$$

can be written in the form (1.1) with

$$
A(\cdot, z, p)=c_{x x}(\cdot, Y(\cdot, p)), \quad B(x, z, p)=\left|\operatorname{det} c_{x, y}(\cdot, Y)\right| f / g^{\circ} Y,
$$

(independent of $z$ ), for elliptic solutions $u$.

The second boundary value problem for the prescribed Jacobian equation (1.3) is to prescribe the image,

$$
T u(\Omega):=Y(\cdot, D u)(\Omega)=\Omega^{*}
$$

and a necessary condition for the existence of an elliptic solution $u$, for which the mapping $T u$ is a diffeomorphism, is the mass balance condition,

$$
\int_{\Omega} f=\int_{\Omega^{*}} g .
$$

For the standard Monge-Ampère equation, $c(x, y)=x \cdot y, Y=p, A=0$, with $T u=D u$, the classical solvability of the second boundary value problem for smooth densities and domains, under the mass balance condition (1.6), was proved by Delanöe, $(n=2)$, [3], Caffarelli [2] and Urbas [22], under the hypothesis that both domains, $\Omega$ and $\Omega^{*}$, are uniformly convex, (in the sense that the principal curvatures of their boundaries are bounded from below by a positive constant). As already pointed out in $[10,11]$, (when $\Omega^{*}$ is a ball), (1.5) implies a nonlinear oblique boundary condition. A weaker interpretation of the boundary condition (1.5) arises through optimal 
transportation, in which case Caffarelli [1] proved that the convexity of the target $\Omega^{*}$ suffices for local smoothness of solutions.

Interest in the general case was stimulated in the last decade through its application to regularity in optimal transportation. In the wake of earlier work on reflector design [27], a condition for local regularity called A3 was found by Ma, Trudinger and Wang in [15] and its degenerate form called A3w was introduced and used for global regularity in [20]. The latter was shown to be sharp by Loeper in [14], utilising the proof in [15] of the necessity of target $c$-convexity. We may write these conditions in the form:

\section{A3 (A3w)}

$$
A_{i j}^{k l} \xi_{i} \xi_{j} \eta_{k} \eta_{l}:=\left(D_{p_{k} p_{l}} A_{i j}\right) \xi_{i} \xi_{j} \eta_{k} \eta_{l}>,(\geq) 0
$$

for all $x, p \in \Omega \times \mathbb{R}^{n}$ such that $Y(x, p) \in \Omega^{*}$ and $\xi, \eta \in \mathbb{R}^{n}$ such that $\xi . \eta=0$. Using the notation,

$$
c_{i j \cdots, k l \cdots}=\frac{\partial}{\partial x_{i}} \frac{\partial}{\partial x_{j}} \cdots \frac{\partial}{\partial y_{k}} \frac{\partial}{\partial y_{l}} \cdots c
$$

and writing $\left[c^{i, j}\right]$ for the inverse of $c_{x, y}=\left[c_{i, j}\right]$, we also have

$$
A_{i j}^{k l}=\left(c_{i j, p q}-c^{r, s} c_{i j, s} c_{r, p q}\right) c^{p, k} c^{q, l},
$$

in accordance with the original formulation in [15]. Note that from (1.7), we see that conditions A3 and A3w are symmetric in $x$ and $y$, while the formulation in terms of $x$ and $p$ shows they are invariant under coordinate transformations in $x$, for fixed $y$.

For global regularity, we also need uniform convexity conditions on our domains, with respect to $c$, as also formulated in [20], namely we assume that $\Omega, \Omega^{*}$ are respectively uniformly $c$-convex, $c^{*}$-convex with respect to each other in the sense that the images $c_{y}(\cdot, y)(\Omega), c_{x}(x, \cdot)\left(\Omega^{*}\right)$ are uniformly convex in $\mathbb{R}^{n}$ for all $y \in \bar{\Omega}^{*}, x \in \bar{\Omega}$.

Theorem 1.1 Let $c \in C^{4}\left(\bar{\Omega} \times \bar{\Omega}^{*}\right)$ be a cost function satisfying conditions A1, A2 and $A 3$ w in $C^{4}$ domains $\Omega, \Omega^{*}$ which are mutually uniformly $c$-convex, $c^{*}$-convex with respect to each other. Suppose $f \in C^{2}(\bar{\Omega}), g \in C^{2}(\bar{\Omega})$ are positive densities satisfying the mass balance condition (1.6). Then there exists an elliptic solution $u \in C^{3}(\bar{\Omega})$ of the second boundary value problem (1.3), (1.5), with Tu a diffeomorphism from $\bar{\Omega}$ to $\bar{\Omega}^{*}$, which is unique up to additive constants.

The corresponding Monge-Kantorovich problem in optimal transportation is to find a measure preserving mapping $T_{0}: \Omega \rightarrow \Omega^{*}$ which maximizes the cost functional

$$
C(T)=\int_{\Omega} f(c(\cdot, T) d x
$$

among all measure preserving mappings $T$ from $\Omega$ to $\Omega^{*}$. A mapping $T: \Omega \rightarrow \Omega^{*}$ is called measure preserving, with respect to densities $f$ and $g$, if it is Borel measurable and for any Borel set $E \subset \Omega^{*}$, 


$$
\int_{T^{-1}(E)} f=\int_{E} g .
$$

The reader is referred to the expositions $[16,23,25,26]$ for further information about optimal transportation.

Corollary 1.2 Under the hypotheses of Theorem 1, there exists a unique diffeomorphism $T=Y(\cdot, D u), \in\left[C^{2}(\bar{\Omega})\right]^{n}$ maximizing the functional $(1.8)$, where $u$ is an elliptic solution of the second boundary value problem (1.3), (1.5).

The solution $u$ of (1.3), (1.5) is called a potential. From elliptic regularity theory it follows that if $c, \Omega, \Omega^{*}, f, g$ are $C^{\infty}$ smooth, then the resultant potentials $u$ and optimal mapping $T$ are also. Note that we have followed the same sign conventions as in [20] but usually the cost functions and potentials are the negatives of those here and the optimal transportation problem is written equivalently as a minimization problem.

These results are proved in [20] under a further hypothesis, namely either the non degenerate condition A3 holds or a global barrier type condition is satisfied in $\Omega$, (called $c$-boundedness in $[17,20]$ ), or the matrix function $A$ depends only on $p$. Such restrictions arise from the second derivative bounds in Section 3 of [20] and do not affect the main examples as presented there in Section 8. The last mentioned alternative sufficient condition follows from a duality argument in Section 3 of [20] which does not seem to extend to general dependence of $A$ on $(x, p)$, (as envisaged in an earlier version of [20]). An alternative sub-solution condition for these second derivative bounds is also given in the recent paper [6]. In the next section we indicate the necessary modifications to obtain the full generality.

We remark also that the results and proofs in [20] were extended to the more general prescribed Jacobian equations in [18] and to parabolic equations in [9].

\section{Second derivative bounds}

Theorem 1.1 follows from a modification to the main second derivative estimate in [20], (also labelled Theorem 1.1). To facilitate the application of the method of continuity from [20], we need to consider more general equations than (1.3), namely we assume still that $A$ is given by (1.4) but consider a general $B>0, \in C^{2}\left(\bar{\Omega} \times \mathbb{R} \times \mathbb{R}^{n}\right)$. We also allow for a domain variation.

Theorem 2.1 Let the matrix function A and domains $\Omega$ and $\Omega^{*}$ satisfy the same conditions as in Theorem 1.1 with the scalar function $B$ as above and let $\Omega_{t}$ and $\Omega_{t}^{*}$ denote $C^{4}$ subdomains of $\Omega$ and $\Omega^{*}$, which are also uniformly $c$ and $c^{*}$-convex with respect to each other. Then any elliptic solution $u \in C^{3}\left(\bar{\Omega}_{t}\right)$ of (1.1), (1.5), for which the mapping Tu is a diffeomorphism from $\Omega_{t}$ to $\Omega_{t}^{*}$, satisfies the a priori estimate

$$
\left|D^{2} u\right| \leq C
$$

where $C$ depends on $c, \Omega_{t}, \Omega_{t}^{*}, B$ and $\sup _{\Omega_{t}}|u|$. 
More specifically, with $c, \Omega$ and $\Omega^{*}$ fixed, the constant $C$ depends on the $C^{2}$ norms and minima of $B$ over compact subsets of $\Omega \times \mathbb{R} \times \mathbb{R}^{n}$ together with the $C^{4}$ smoothness of the domains $\Omega_{t}$ and $\Omega_{t}^{*}$ and their uniform convexity constants. We also observe the simple gradient estimate

$$
|D u|=\left|c_{x}(\cdot, T u)\right| \leq \sup _{\Omega \times \Omega^{*}}\left|c_{x}\right|
$$

Proof From [20] it suffices to derive a global bound for $D^{2} u$ in terms of its boundary trace as that is subsequently estimated in Section 4 of [20], using the obliqueness estimate from Sect. 2. First we note from [20] that the uniform $c$-convexity of $\Omega$ implies the existence of a $C^{2}$ defining function $\phi$ for $\Omega$ satisfying

$$
\left[D_{i j} \phi-c^{l, k} c_{i j, l}(\cdot, T u) D_{k} \phi\right] \xi_{i} \xi_{j} \geq \delta_{0}|\xi|^{2}
$$

in a fixed neighbourhood $\mathcal{N}$ of $\partial \Omega$, for all $\xi \in \mathbb{R}^{n}$ and for a fixed constant $\delta_{0}>0$. Noting also the formula,

$$
D_{p_{k}} A_{i j}(\cdot, D u)=c^{l, k} c_{i j, l}(x, T u),
$$

we have from the global second derivative estimate, Theorem 3.1 in [20],

$$
\sup _{\mathcal{N}}\left|D^{2} u\right| \leq C\left(1+\sup _{\partial \mathcal{N}}\left|D^{2} u\right|\right)
$$

with constant $C$ depending on $c, \Omega, \Omega^{*}, B$ and $\sup (|u|+|D u|)$. Consequently the global estimation is reduced to an interior estimate in a strictly contained subdomain $\Omega^{\prime}=\Omega-\overline{\mathcal{N}}$.

Interior second order derivative estimates for solutions of Monge-Ampère type equations, under the weak condition $\mathrm{A} 3 \mathrm{w}$, are derived in [12] and [13]. We need to apply these in $c$-sections of $u$ whose diameters will be controlled by the strict convexity results in [4] and [24]. First we note that the ellipticity condition (1.2) and the $c$-convexity of the domain $\Omega$ implies that $u$ is $c$-convex in $\Omega$ [21], (see also [20] and [8]), that is for any $x_{0} \in \Omega$ and $y_{0}=T u\left(x_{0}\right)$, we have

$$
u(x) \geq u\left(x_{0}\right)+c\left(x, y_{0}\right)-c\left(x_{0}, y_{0}\right)
$$

for all $x \in \Omega$. The $c$-section, $S_{h}\left(x_{0}\right)$ for $h>0$ is then defined by

$$
S_{h}\left(x_{0}\right)=\left\{x \in \Omega \mid u(x)<u\left(x_{0}\right)+c\left(x, y_{0}\right)-c\left(x_{0}, y_{0}\right)+h\right\}
$$

Writing $b=B(\cdot, u, D u) /\left|\operatorname{det} c_{x, y}(\cdot, T u)\right|$, it follows since $\mathrm{u}$ is c-convex and Tu a diffeomorphism, that $\mathrm{u}$ is a potential for the corresponding Monge-Kantorovich problem with densities $f=b$ and $g=1$. Furthermore there exist positive constants $b_{0}$ and $b_{1}$, depending on $B,\left|\operatorname{det} c_{x, y}\right|$ and $\sup (|u|+\mid D u)$ such that $b_{0} \leq b \leq b_{1}$. We 
now claim that, from [4] and [24], we have a strict convexity estimate, that is for any $R<\operatorname{dist}\left(x_{0}, \partial \Omega_{t}\right)$, there exists $h>0$, depending on $c, \Omega, \Omega^{*}$ and $b_{0}, b_{1}$, such that

$$
S_{h}\left(x_{0}\right) \subset B_{R}\left(x_{0}\right)
$$

where $B_{R}=B_{R}\left(x_{0}\right)$ denotes the ball of radius $R$ and centre at $x_{0}$. This follows in a standard way by contradiction for otherwise there would exist a sequence of potentials converging uniformly to a $c$-convex potential of a limiting problem with densities bounded away from 0 and $\infty$ which would not be strictly $c$-convex at $x_{0}$, thereby contradicting the strict convexity assertions in [4] and [24]. We also note that a crucial hypotheses in these works is the uniform $c$-convexity of the target domain which is satisfied by hypothesis in our case. So that there is no confusion, we note also that uniform convexity in our terminology, as employed for example in [7], is designated strong in [4], (in accordance with usage in convexity theory where uniform can mean a uniform modulus of convexity).

To complete the proof of Theorem 2.1, we then note that for sufficiently small $R$ there exists a smooth defining function $\phi$ satisfying (2.3) in $B_{R}$ so that we can apply the interior second derivative estimate in [12] in $S_{h}\left(x_{0}\right)$ to obtain an estimate

$$
\sup _{B_{\rho}}\left|D^{2} u\right| \leq C
$$

for sufficiently small $\rho$ depending on $h$ and $\sup \mid D\left(u-c\left(\cdot, y_{0}\right) \mid\right.$. By using a finite covering the estimate (2.8) is extended to $\Omega^{\prime}$ and we conclude Theorem 2.1, with the asserted constant dependence, through replacement of $\Omega$ and $\Omega^{*}$ by $\Omega_{t}$ and $\Omega_{t}^{*}$ respectively.

We remark that a stronger result is proved in [4] than that used above, namely that the optimal mappings are strictly convex and Hölder continuous whereas in [24] only strict convexity and continuity is proved, (using an earlier version of [4] which assumes condition $\mathrm{A} 3 \mathrm{w}$ without the orthogonality restriction on $\xi$ and $\eta$ ). Note also that in two dimensions the strict convexity was already proved in [5] under a one-sided density bound as an extension of the classical result of Aleksandrov for the Monge-Ampère equation, (see for example [19]). However the argument in [5] also depends on a result established in [4]. We remark also that when the strong condition A3 holds, then the proof of the second derivative estimate is much simpler and already follows from [15]; see also [19].

From the estimates (2.1),(2.2), Theorem 1.1 follows immediately by using Theorem 2.1, (instead of Theorem 1.1 in [20]), in the method of continuity argument presented in Section 5 of [20].

Open Access This article is distributed under the terms of the Creative Commons Attribution License which permits any use, distribution, and reproduction in any medium, provided the original author(s) and the source are credited. 


\section{References}

1. Caffarelli, L.: The regularity of mappings with a convex potential. J. Am. Math. Soc. 5, 99-104 (1992)

2. Caffarelli, L.: Boundary regularity of maps with convex potentials II. Ann. Math. 144, 453-496 (1996)

3. Delanoë, Ph.: Classical solvability in dimension two of the second boundary value problem associated with the Monge-Ampère operator. Ann. Inst. Henri Poincaré Analyse Non Linéaire 8, 443-457 (1991)

4. Figalli, A., Kim, Y.-H., McCann, R.J.: Hölder continuity and injectivity of optimal maps. Arch. Rat. Mech. Anal. 209, 747-795 (2013)

5. Figalli, A., Loeper, G.: $C^{1}$ regularity of solutions of the Monge-Ampère equation for optimal transport in dimension two. Calc. Var. Partial Differential Equations 35, 537-550 (2009)

6. Jiang, F., Trudinger, N.S., Yang, X.-P.: On the Dirichlet Problem for Monge-Ampère type equations. Calc. Var. Partial Differ. Equ. (to appear). doi:10.1007/s00526-013-0619-3

7. Gilbarg, D., Trudinger, N.S.: Elliptic partial differential equations of second order. Springer, Berlin (1983)

8. Kim, Y.-H., McCann, R.J.: Continuity, curvature and the general covariance of optimal transportation. J. Eur. Math. Soc. 12, 1009-1040 (2010)

9. Kitagawa, J.: A parabolic flow towards solutions of the optimal transportation problem on domains with boundary. J. Reine Angew. Math. 672, 127-160 (2012)

10. Lions, P.-L., Trudinger, N.S., Urbas, J.: Neumann problem for equations of Monge-Ampère type, Comm. Pure Appl. Math. 39, 539-563 (1985)

11. Lions, P.-L., Trudinger, N.S., Urbas, J.: Neumann problem for equations of Monge-Ampère type. Proc. Centre Math. Anal. 10, 135-140 (1985)

12. Liu, J., Trudinger, N.: On Pogorelov estimates for Monge-Ampere type equations. Discret. Contin. Dyn. Sys. 28, 1121-1135 (2010)

13. Liu, J., Trudinger, N.S., Wang, X.-J.: Interior $C^{2, \alpha}$ regularity for potential functions in optimal transportation. Comm. Partial Differ. Equ. 35, 165-184 (2010)

14. Loeper, G.: On the regularity of solutions of optimal transportation problems. Acta Math. 202, 241-283 (2009)

15. Ma, X.-N., Trudinger, N.S., Wang, X.-J.: Regularity of potential functions of the optimal transportation problem. Arch. Rat. Mech. Anal. 177, 151-183 (2005)

16. Rachev, S.T., Ruschendorff, L.: Mass transportation problems. Springer, Berlin (1998)

17. Trudinger, N.S.: Recent developments in elliptic partial differential equations of Monge-Ampère type. Int. Congress Math. III, 291-301 (2006, Eur. Math. Soc., Zürich)

18. Trudinger. N.S.: On the prescribed Jacobian equation, Gakuto Intl. Series, Math. Sci. Appl. 20 (2008), Proc. Intl. Conf. for the 25th Anniversary of Viscosity, Solutions, 243-255

19. Trudinger, N.S., Wang, X.-J.: The Monge-Ampère equation and its geometric applications. In: Handbook of geometric analysis. International Press, USA, pp. 467-524 (2008)

20. Trudinger, N.S., Wang, X.J.: On the second boundary value problem for Monge-Ampère type equations and optimal transportation. Ann. Scuola Norm. Sup. Pisa Cl. Sci. Ser. 5 8, 143-174 (2009)

21. Trudinger, N.S., Wang, X.-J.: On strict convexity and continuous differentiability of potential functions in optimal transportation. Arch. Rat. Mech. Ȧnal. 192, 403-418 (2009)

22. Urbas, J.: On the second boundary value problem for equations of Monge-Ampère type. J. Reine Angew. Math. 487, 115-124 (1997)

23. Urbas, J.: Mass transfer problems. University of Bonn, Lecture Notes (1998)

24. Vetois, J.: Continuity and injectivity of optimal maps, preprint (2011)

25. Villani, C.: Topics in optimal transportation. Amer. Math. Soc. (2003)

26. Villani, C.: Optimal transportation, old and new. Springer, Berlin (2008)

27. Wang, X.-J.: On the design of a reflector antenna. Inverse Prob. 12, 351-375 (1996) 